\title{
BSPD and FDS invest in exceptional researchers
}

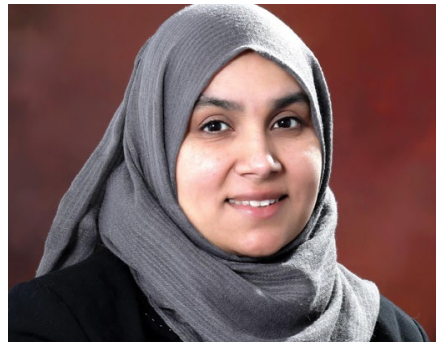

Armaana Ahmad

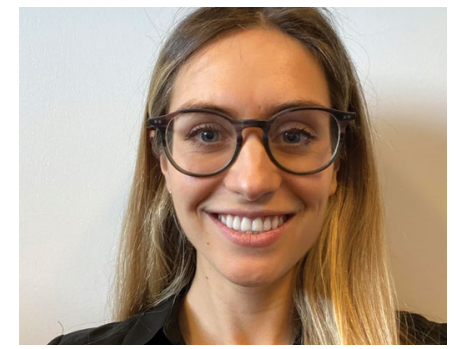

Julia Palmer

Two exceptional research proposals focusing on the needs of young patients have been awarded grants jointly by the British Society of Paediatric Dentistry (BSPD) and the Faculty of Dental Surgery of the Royal College of Surgeons (England) (FDS). One proposal is for research into jaw pain in young people and the other a feasibility study for genetic testing of children with a rare developmental condition.

The two successful BSPD members in 2021 are Julia Palmer of Newcastle Dental School who was awarded $£ 5,272$, and Armaana Ahmad of UCL Dental Institute, who was awarded $£ 9,297.88$.

The joint FDS/BSPD pump-priming grants are awarded annually and are worth up to $£ 10,000$ each.

Prominent BSPD member and Chair of the FDS Research Committee, Professor Helen Rodd, said: 'We were overwhelmed by the quality of the research proposals this year. The two winning proposals were exceptional in terms of the benefit to patients. Both were ranked highly by five reviewers from different dental specialties.

'These grants don't just support the research but the individual researchers too who have shown great promise and whose academic careers will benefit from the recognition that such a prestigious grant confers.'

The funding received by Julia will help fund her research into understanding the experience and needs of young people who have temporomandibular disorder (TMD), or jaw pain. There is currently no research relating to adolescent experience of TMD and young people with the condition are managed in the same way as adults.

Since there has not been any qualitative research among under $18 \mathrm{~s}$, the pump-priming funding will help her set up focus groups comprised of patients to understand their concerns and needs. She will also undertake qualitative research with dentists in primary and secondary care who treat these adolescent patients and devise a questionnaire to get a picture of how the condition is managed and whether prevalence is increasing.

Armaana Ahmad's proposal is a feasibility study examining genetic investigations for children who have type II dentinogenesis imperfecta (DI).

There are two main types of DI, one is part of a medical condition affecting teeth and bones, osteogenesis imperfecta, better known as brittle bone disease. The other main type manifests as a dental condition featuring discoloured teeth which are weak and prone to fracture.

Her research interest has been inspired by her clinical care of patients who have the inherited condition. Children who appear to have only dental symptoms and no bone symptoms are not currently able to have genetic testing to confirm which type of DI they have. $\diamond$ 